\title{
SISTEMA DE RIEGO AUTÓNOMO PARA PEQUEÑOS CULTIVOS BASADO EN MEDICIÓN DE TEMPERATURA Y HUMEDAD
}

\author{
AUTONOMOUS IRRIGATION SYSTEM FOR SMALL CROPS BASED ON TEMPERATURE AND SOIL \\ HUMIDITY MESURE
}

\author{
Pedro Fernando Martín Gómez ${ }^{1}$, Jairo Orlando Montoya Gómez ${ }^{2}$, Guillermo Enrique Muñoz Serrano ${ }^{3}$ \\ 1 Pedro Fernando Martín Gómez, Ph.D., Profesor Asociado, Universidad de La Salle, \\ pmartin@unisalle.edu.co. \\ 2 Jairo Orlando Montoya Gómez, M.Sc., Profesor Asociado, Universidad de La Salle, \\ jamontoya@unisalle.edu.co. \\ ${ }^{3}$ Guillermo Enrique Muñoz Serrano, Ing., Universidad de La Salle, balrogasimov@yahoo.com.
}

\section{RESUMEN}

Se describe el desarrollo de un sistema de riego autónomo para un cultivo de maíz de $2400 \mathrm{~m}^{2}$, ubicado en la finca La Reforma, Colombia. Este sistema fue desarrollado buscando optimizar el uso del recurso hídrico proveniente de una quebrada cercana a la zona del cultivo, la cual es compartida con otros cultivadores. El propósito es eliminar daños por aplastamiento y fractura de las plantas, pérdidas por falta de irrigación, costos adicionales por mano de obra y desperdicio de agua, debido a que el agua se utiliza solo cuando es necesaria, lo que evita la erosión por sobre riego y el desabastecimiento de otros cultivos. Adicionalmente, se pretendía implementar un sistema simple y económico para pequeños agricultores, pero a su vez, funcional, con tecnología disponible en el mercado nacional. La solución se compone de un sistema de aspersión, con el menor número de elementos para mayor cubrimiento de área, el cual se acciona automáticamente a partir de la medición de temperatura ambiente y humedad del suelo. Se implementó un sistema básico modular, al cual se le puedan adicionar posteriormente otras variables y se validó en un el cultivo de maíz durante seis meses, a partir de la distribución equitativa de seis sensores de humedad del suelo y de temperatura, dentro del área de cultivo; los cuales interactúan con seis aspersores 70-10-30G para cubrir 20 m de diámetro a 72 psi cada uno, accionado por un microcontrolador en un circuito electrónico diseñado. Se presentan resultados sobre la cosecha final mejorando las condiciones físicas de color, aspecto y presentación más fresca y homogénea; con menor consumo de agua frente al uso habitual de suministro permanente con manguera y condiciones de las plantas más verdes y productivas en general; se obtuvo una producción equivalente a seis veces más de maíz, con respecto a la producción normal, para el mismo tamaño de lote; con mejores condiciones de calidad, mediante un sistema sencillo y de bajo costo, cuya recuperación de inversión se obtiene en un tiempo de dos a tres cosechas.

Palabras clave: Riego; aspersión; sistema autónomo; cultivo de maíz; sistema automático.

Recibido: 16 de Junio de 2017.

Received: June 16, 2017.
Aceptado: 08 de Septiembre de 2017.

Accepted: September 8th, 2017. 


\begin{abstract}
The development of an autonomous irrigation system for a corn crop of $2400 \mathrm{~m}^{2}$, located in the farm La Reforma, Colombia, is described. This system was developed to optimize the use of the water resource coming from a ravine near stream close to the crop, which is shared with other growers. The purpose is to eliminate damage by crushing and fracture of the plants, losses due to lack of irrigation, additional costs for labor and waste of water, because it is only used when it is necessary, which avoids erosion by over-irrigation and shortage of other crops. In addition, it was intended to implement a simple and economic system for small farmers, but at the same time, functional with technology available in the national market. The solution consists of a sprinkler system, with the least number of elements to cover the largest area, which is automatically activated from the measurement of ambient temperature and soil moisture. A modular basic system was implemented, which could be later added to other variables and validated in a corn crop for six months, from the equitable distribution of six soil moisture and temperature sensors, within the crops area; Which interact with six 70-10-30G sprinklers to cover $20 \mathrm{~m}$ in diameter at 72 psi each, powered by a microcontroller in a designed electronic circuit. Results are presented on the final harvest, improving the physical conditions of color, appearance and presentation more fresh and homogeneous; With lower water consumption compared to the usual use of permanent supply with hose and conditions of the greenest and most productive plants in general; A production equivalent to six times more corn was obtained, compared to normal production, for the same batch size; With better quality conditions, through a simple and low-cost system, whose recovery of investment is obtained in a time of two to three harvests.
\end{abstract}

Keywords: Irrigation; sprinkler; autonomous system; corn crop; automatic system.

Cómo citar este artículo: P. F. Martín-Gómez, J. O. Montoya-Gómez, G. E. Muñoz-Serrano, "Sistema de riego autónomo para pequeños cultivos basado en medición de temperatura y humedad," Revista Politécnica, vol. 13, no. 25, pp. 65-74, 2017. 


\section{INTRODUCCIÓN}

Un sistema autónomo en agricultura ayuda al desarrollo del cultivo sin la presencia permanente del cultivador, permitiendo controlar un área más grande de cultivo, junto con los beneficios adicionales que trae para el consumo, el uso y el menor desperdicio de fertilizantes y de agua, sumado al mejor rendimiento del cultivo con mejores características del producto. Esta tendencia propone actualmente un concepto vital para ser más productivo y competitivo en el mercado. Es por esta razón, que la agricultura ha tomado una gran relevancia en los últimos años, con investigación desde diferentes frentes, para obtener un alto grado de producción, impactando lo mínimo posible el suelo y el medio ambiente. Lo anterior, se consigue mediante la implementación de tecnología para la producción, planeación y gestión automatizada de los cultivos en lo que se denomina como "Agricultura de Precisión" como lo describe Blackmore [1]. De esta manera, se puede obtener el máximo potencial por unidad de área, generando ahorro en insumos, menor degradación del suelo, rendimientos óptimos en las cosechas y menor costo de producción, a partir del uso de equipos automáticos basados en desarrollo de sensórica especializada por infra rojo [2] y sistemas de detección por visión [3], tecnologías de posicionamiento global (GPS) para control vehicular [4] y detección de límites de campo [5], sistemas de información geográfica (GIS) para distribución del suelo [6] y aplicaciones robotizadas para siembra [7], suministro de fertilizantes [8], comunicación inalámbrica para irrigación [9], irrigación por aspersión de sólidos [10] y líquidos [11] y desmalezado [12] entre otros. Estos sistemas basados en tecnologías capaces de hacer un mejor manejo del cultivo a partir de sus condiciones químicas, físicas y geográficas con sistemas computarizados; para hacer un adecuado cubrimiento del terreno y logrando a la vez mayor productividad en forma localizada, con la variación punto a punto en la aplicación de insumos. En aplicaciones sobre maíz, hay sistemas de riego recientes dependiendo de la variabilidad meteorológica sobre la distribución del agua en sistemas de pivote central [13], un prototipo de programador de riego [14] y en planificación y control de riego basado en humedad del suelo [15].

El objetivo del presente trabajo era desarrollar un sistema automático de riego para un cultivo de maíz, buscando mayor aprovechamiento del recurso hídrico de difícil disponibilidad en la región; cuyo propósito fundamental era eliminar los daños por aplastamiento y fractura de las plantas debidos al movimiento de mangueras de riego, las pérdidas por falta de irrigación en varias áreas, los costos adicionales por mano de obra evitando desperdicios de agua, gracias al consumo estrictamente necesario y evitar la erosión del suelo y el desabastecimiento de otros cultivos por desvío de afluentes hídricos.

El proyecto se llevó a cabo en la finca la Reforma, del municipio de Machetá, Cundinamarca; sobre un cultivo de maíz tipo porva, tomado como cultivo piloto para la implementación del sistema de riego autónomo [16]. El sistema se activa por medio de sensores ubicados en terreno que envían las lecturas de humedad relativa y temperatura a un sistema de adquisición de datos para la toma de decisiones, activando o desactivando los actuadores del sistema de aspersión. También se desarrolló un sistema de información básico, que sirvió como plataforma para que el usuario conociera el desempeño del sistema.

El sistema diseñado es flexible y permite la expansión para facilitar en un futuro la implementación del sistema de dosificación de fertilizantes al suelo, con manejo independiente de los tanques, y la dosis ajustada a los parámetros requeridos en cada punto del recorrido; garantizando las concentraciones de cada nutriente, mediante la aplicación de acciones de control simultáneas.

\section{MATERIALES Y MÉTODO}

El sistema desarrollado emplea elementos comerciales disponibles en el mercado local, tales como: sensores de humedad del suelo y temperatura ambiente, filtros, aspersores y electroválvulas, microcontroladores, motobomba eléctrica y elementos electrónicos básicos junto con el sistema de mangueras y tuberías de distribución.

La metodología seguida se describe a continuación:

Se determina el tiempo de riego que depende de las condiciones de flujo del afluente disponible y de las cantidades demandadas por el cultivo de maíz específicamente. Se hizo primero el cálculo de caudal del acuífero mediante el método de flotador 
y señaladores de distancia, calculando el tiempo de transporte del flotador entre las marcas y el área transversal aproximada del afluente en un sitio aguas arriba, considerado apropiado para instalar la toma de agua del sistema (Fig. 1). De esta forma se obtuvo una velocidad promedio de $0,2157 \mathrm{~m} / \mathrm{s}$ o un caudal equivalente de $0,009 \mathrm{~m}^{3} / \mathrm{s}$. Una vez dimensionado un reservorio de $0,21 \mathrm{~m}^{3}$ sobre la quebrada fue calculada la pérdida de presión en los ductos, considerando una distancia aproximada de $300 \mathrm{~m}$ entre la quebrada y la finca y las condiciones geográficas específicas.

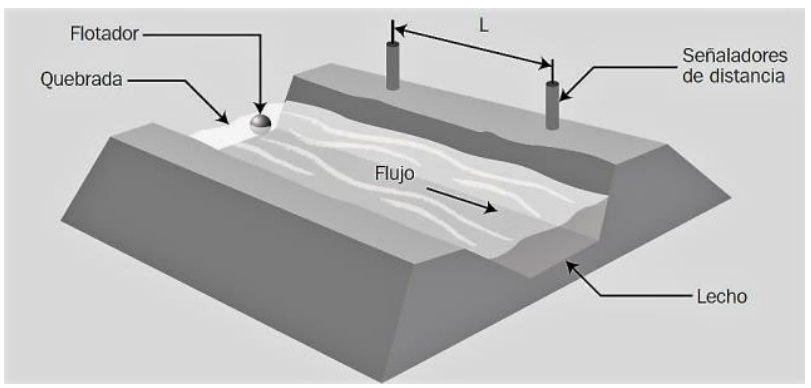

Fig. 1. Cálculo de caudales por método de marcadores. Fuente: Manual práctico para el diseño de sistemas de mini riego. Programa Especial para la Seguridad Alimentaria (PESA). Gobierno de Honduras.

Luego, conociendo la cantidad máxima disponible de agua en un instante y el volumen del pozo, se calculó el caudal de la manguera sobre el punto de toma del agua a una altura de $50 \mathrm{~m}$ por encima de la finca dando un valor de $0,01585 \mathrm{~m}^{3} / \mathrm{s}$. Con el caudal disponible fue dimensionado el sistema de aspersión y seleccionados los componentes para aspersores radiales 70-10-30G de marca intecmecol de 1 pulgada de diámetro.

El cálculo del porcentaje de humedad fue el punto de partida para la automatización del riego, ya que representa el exceso o falta de agua durante las diferentes etapas del cultivo y puede causar la pérdida total o parcial de la cosecha. El suelo en la finca es un suelo franco arcilloso con densidad entre 1.0 a $1.4 \mathrm{~g} / \mathrm{cm}^{3}$ que, combinada con la lámina de agua en los diferentes tiempos del cultivo, brinda el porcentaje de humedad en suelo requerido en cada etapa. Para ello se tuvieron en cuenta factores como: el tipo de suelo, la lámina de agua a aplicar y la profundidad de la lámina; basados en el consumo de agua del cultivo de maíz en sus diferentes etapas, como se muestra en Tabla 1.

Debido a que las raíces del maíz son superficiales, los sensores se ubicaron a $0,1 \mathrm{~m}$ de profundidad que es donde en promedio hay un mayor número de raíces y por lo tanto el sector de la planta donde hay mayor absorción de agua y nutrientes. Con estos datos fueron calculadas las cantidades de lámina de agua diaria en cada etapa, de acuerdo con la Tabla 1.

Tabla 1. Porcentaje de humedad con respecto a la etapa del cultivo.

\begin{tabular}{lcccc}
\hline Periodo & Semana & $\begin{array}{c}\text { Lámina } \\
\text { Agua } \\
\text { [mm/día] }\end{array}$ & Días & $\begin{array}{c}\text { Humedad } \\
{[\%]}\end{array}$ \\
\hline Siembra & 0 & 1 & 7 & 5 \\
Brote & $1-2$ & 1.5 & 7 & 7,5 \\
4-5 hojas & $3-4$ & 2 & 7 & 10 \\
6-7 hojas & $5-7$ & 3 & 7 & 15 \\
9-10hojas & $8-9$ & 4 & 7 & 20 \\
Floración & $10-13$ & 7 & 7 & 35 \\
Llenado & $14-18$ & 5,5 & 7 & 27,5 \\
Madurez & $18-21$ & 4 & 7 & 20 \\
Secado & $22-24$ & 0 & 7 & 0 \\
\hline
\end{tabular}

Fuente: [13]. Muñoz, G., 2015.

Para la medición fueron seleccionados sensores de humedad SEN 0114 cuyas características se muestran en la Tabla 2, y fueron caracterizados de acuerdo con la curva de la Fig. 2.

Tabla 2. Especificaciones del sensor de humedad SEN0114.

\begin{tabular}{lc}
\hline \multicolumn{1}{c}{ Característica } & Valor \\
\hline Tensión de alimentación & 3,3 a 5,0V \\
Tensión de salida & $0-2,3 \mathrm{~V}$ \\
Corriente & Max. 45mA \\
Vida útil & Aprox. 1 año \\
Largo & $60 \mathrm{~mm}$ \\
Ancho & $20 \mathrm{~mm}$ \\
Espesor & $5 \mathrm{~mm}$ \\
\hline
\end{tabular}

Fuente: http://www.dfrobot.com.cn/index.php

Para estructurar el sistema de distribución y aplicación sobre el cultivo, se hizo un análisis teniendo en cuenta el diámetro de aspersión del fluido de acuerdo con la Fig. 3 y las dimensiones del cultivo de $30 \mathrm{~m}$ por $80 \mathrm{~m}$, con una distribución 
de 6 aspersores para el cubrimiento máximo. De acuerdo con las pruebas preliminares los aspersores 70-10-30G tienen un diámetro de cubrimiento de $20 \mathrm{~m}$ con 72 psi de presión, seleccionando la segunda alternativa de la Fig. 3, ya que presenta menos zonas de sobre irrigación y las áreas sin riego son muy pequeñas. De esta forma se eliminaron los problemas de rupturas de plantas por traslado de manguera con seis puntos de aspersión estáticos activados cada uno por válvulas en forma secuencial, con una limitante de máximo dos válvulas simultáneas por restricción del caudal de la quebrada fuente, ya que, al abrir más de dos válvulas al tiempo, el diámetro de cubrimiento disminuyó a $8 \mathrm{~m}$.

\section{Sensor de Humedad}

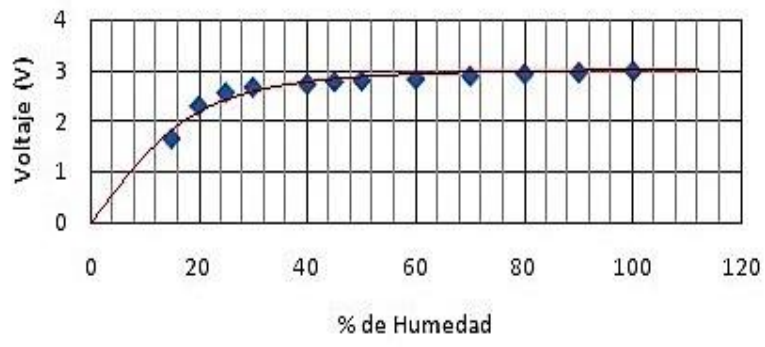

Fig. 2. Curva de calibración del sensor de humedad SEN0114. Fuente: [13]. Muñoz, G., 2015.

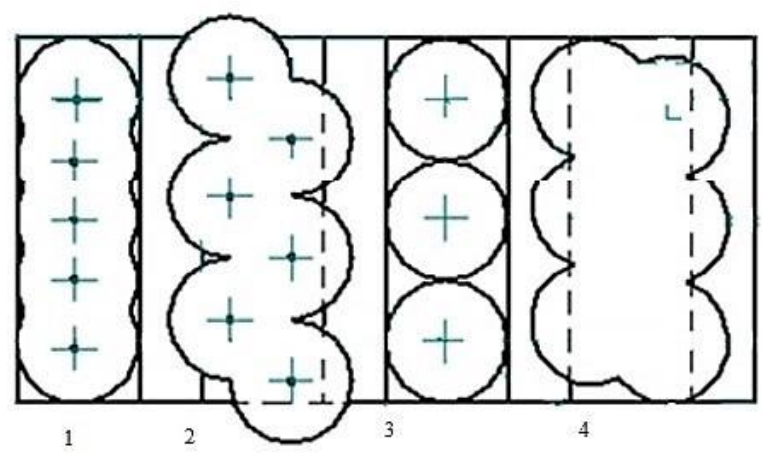

Fig. 3. Alternativas de distribución de los aspersores en el cultivo para el máximo cubrimiento. Fuente: [13]. Muñoz, G., 2015.

Luego de los cálculos, fue realizada la instalación en campo. Para las pruebas preliminares, fueron instalados los aspersores con tubería de PVC asegurada sobre postes de madera aproximadamente a $2 \mathrm{~m}$ de altura, considerando la altura de las plantas de maíz, como se observa en la Fig. 4; y luego fueron remplazadas las válvulas manuales por electroválvulas tipo ROB-10456 de bajo costo. En la Fig. 5, se muestra una prueba preliminar de funcionamiento del sistema con un aspersor.

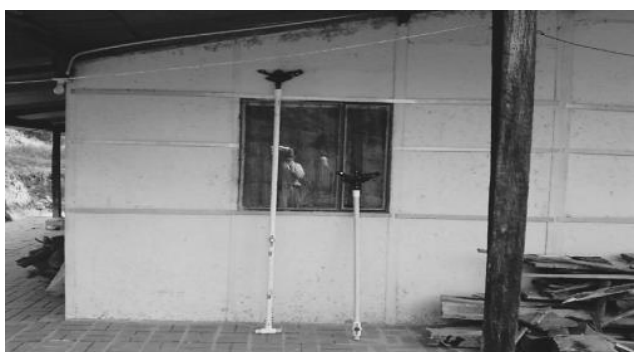

Fig. 4. Estructuras de soporte de los aspersores para pruebas preliminares con válvula manual. Fuente: [13]. Muñoz, G., 2015.

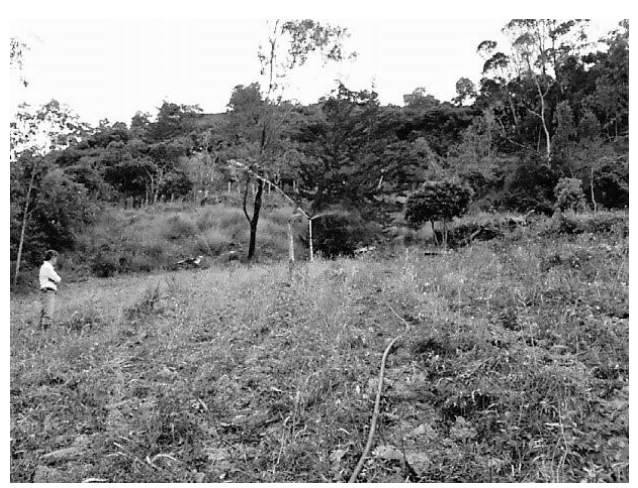

Fig. 5. Prueba preliminar de funcionamiento del sistema de riego con un aspersor. Fuente: [13]. Muñoz, G., 2015.

Con el fin de automatizar el accionamiento del sistema de bombeo, se programó el sistema de control con microcontrolador a partir de la lectura de temperatura y humedad. Para el funcionamiento del sistema, se toman las lecturas de los sensores, se graban en un archivo *.txt que sirve como archivo histórico de referencia.

El programa para la adquisición de datos se desarrolló en Dev $\mathrm{C}++$ de Bloodshed Software, un compilador de $\mathrm{C}++$ con licencia GNU; y los datos son recibidos por medio de una conexión serial RS232 entre el computador y el micro controlador PIC 18F4550 en forma unidireccional, ya que el PIC solo envía datos; por lo cual el sistema puede seguir funcionando así el computador este apagado o no encuentre conexión. Este PIC 18F4550, es económico y muy comercial, lo que permite encontrar el repuesto fácilmente. Tiene 13 entradas 
análogas lo que facilita con una modificación del código para recibir hasta 13 señales análogas de sensores, además de conexión USB para una futura actualización del sistema al buscar una comunicación serial USB y no la RS232 utilizada actualmente como se muestra en la Fig. 6.

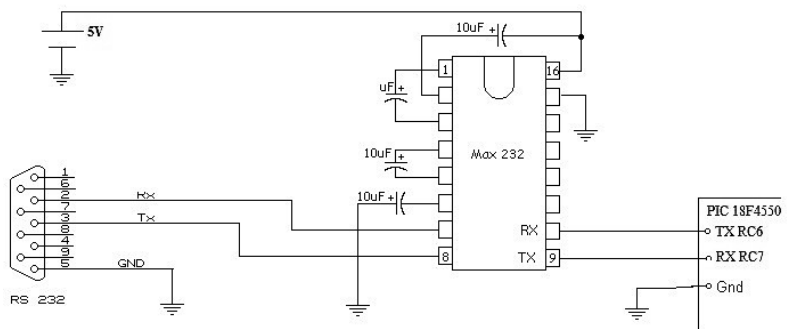

Fig. 6. Conexión del PIC18F4550 al computador por medio de un MAX232. Fuente: [13]. Muñoz, G., 2015.

En la Fig. 7, el diagrama de flujo del programa del PC.

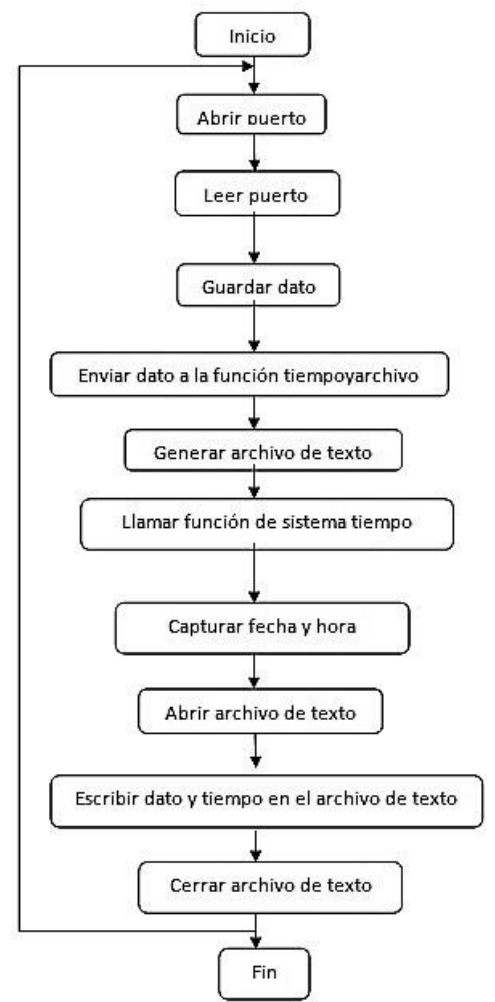

Fig. 7. Diagrama de flujo del programa de adquisición en el PC para guardar datos de humedad en archivo *.txt. Fuente: [13]. Muñoz, G., 2015.
El programa de control desarrollado en el microcontrolador fue programado en SDCC que tiene licencia GNU para flujo de agua constante de la quebrada y las válvulas de cada aspersor por accionamiento on-off ya que en el riego se requiere de todo el caudal disponible para hacer la tarea de forma rápida y eficiente. El diagrama de bloques del sistema de control se muestra en la Fig. 8.

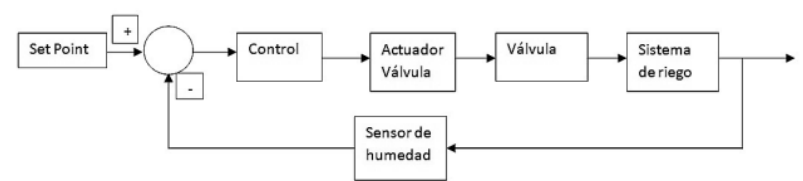

Fig. 8. Diagrama de bloques del Sistema de control de accionamiento del riego por aspersión. Fuente: [13]. Muñoz, G., 2015.

Para iniciar el control se tuvo en cuenta el porcentaje de humedad con respecto al tiempo a partir de la Tabla 1, que muestra los "set points" de acuerdo con la etapa de cultivo, a través del conversor análogo digital del PIC que tiene una resolución de 8 bits y retorna un número entre 0 y 255 según el caso, ver Tabla 3.

Tabla 3. Relación entre el porcentaje de humedad y el voltaje, y su valor equivalente entre 0 y 255.

\begin{tabular}{ccc} 
Humedad, (\%) & Voltaje, (V) & $\begin{array}{c}\text { Equivalencia } \\
0-255 \\
\text { (Set point) }\end{array}$ \\
\hline 5 & 1,493924786 & 76 \\
7.5 & 1,71409234 & 87 \\
10 & 1,870303705 & 95 \\
15 & 2,090471259 & 107 \\
20 & 2,246682625 & 115 \\
35 & 2,550553997 & 130 \\
27.5 & 2,419603001 & 123 \\
20 & 2,246682625 & 115 \\
0 & 0 & 0
\end{tabular}

Fuente: [13]. Muñoz, G., 2015.

El circuito electrónico del sistema consta de 6 sensores de humedad, uno por cada aspersor; los cuales adquieren la señal de humedad en tierra y la envían al microcontrolador PIC 17F4550, el cual evalúa el valor con el fin de determinar si se requiere o no el riego. En caso de ser necesario el 
riego, activa el aspersor del sector respectivo mediante una de las 6 salidas del puerto B; cuya señal es enviada a uno de los 6 opto acopladores, activando el transistor de potencia asociado, y a su vez dispara la electroválvula respectiva, permitiendo el paso del caudal de agua hacia el aspersor para el riego del sector. Si se presenta el caso en que más de un área requiere riego, el sistema compara los niveles de humedad de las áreas y selecciona el menor para iniciar el riego. (Fig. 9).

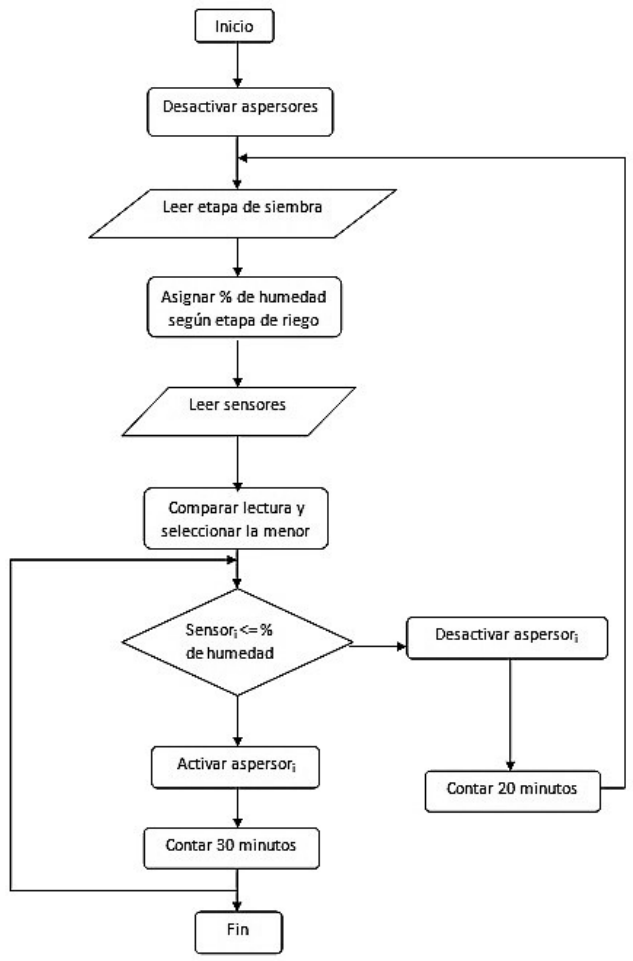

Fig. 9. Diagrama de Flujo de accionamiento del sistema. Fuente: [13]. Muñoz, G., 2015.

\section{RESULTADOS}

El sistema fue construido en la Finca La Reforma y puesto en funcionamiento durante el período de un cultivo de maíz de aproximadamente 6 meses en forma automática con los siguientes resultados:

El sistema de riego autónomo mostró grandes prestaciones al usuario final, con un circuito de riego estable y fijo eliminando las pérdidas causadas por aplastamiento, así como los costos de mano de obra por traslado del aspersor; permitiendo un aumento de tiempo disponible para desarrollar otras labores dentro del cultivo.

El riego controlado permitió un aumento significativo de la producción, ya que el cultivo anterior del mismo producto en un área de $6400 \mathrm{~m}^{2}$ generó una producción total de 5 cargas de maíz de $100 \mathrm{~kg}$ cada una y el resultado en el cultivo prototipo fue de 11 cargas, en tan solo $2400 \mathrm{~m}^{2}$; lo que significa proporcionalmente una producción 5,87 veces mayor.

Las ventajas del sistema no solo radican en la eficiencia en el riego y la desaparición de pérdidas por aplastamiento que llegaban casi a un 35\% de las plantas sembradas, sino que el tiempo que se gastaba en el traslado de la manguera ahora queda disponible para otros trabajos asociados con el cultivo en cuestión (Fumigación, abonado y deshierbe).

La Fig. 10, muestra en un cultivo anterior, los problemas de maleza fuera del sector del medio donde las plantas de maíz son casi inexistentes y el atraso por falta de riego en las primeras etapas del desarrollo; donde el pasto crece con mayor rapidez que el maíz, retrasando aún más su crecimiento y generando abandono del sector.

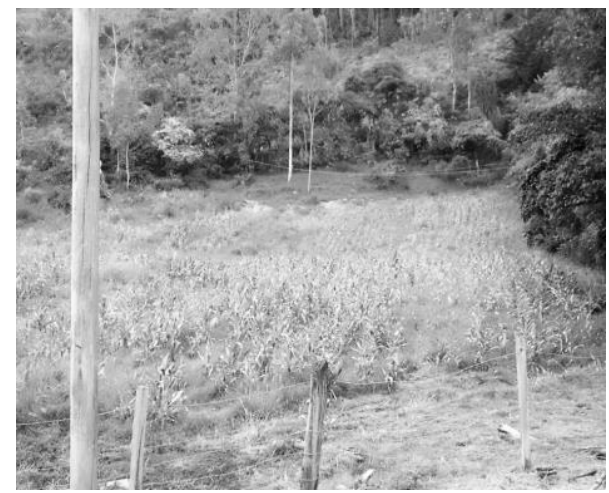

Fig. 10. Cultivo de maíz anterior al desarrollo del sistema de riego, con problemas de crecimiento de malezas y falta de riego. Fuente: [13]. Muñoz, G., 2015.

La Fig. 11, muestra el aspecto general del cultivo con el sistema de riego autónomo a los 2 meses, con mejor aspecto físico, mejor color y una buena distribución y mantenimiento del área de cultivo. 


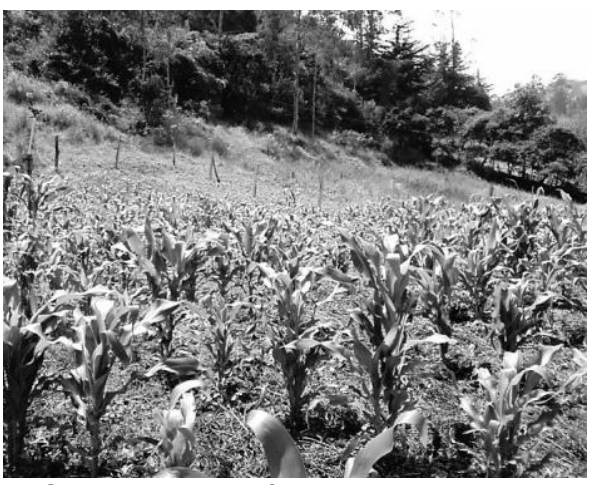

Fig. 11. Cultivo de maíz con el sistema de riego desarrollado, de mejor crecimiento y aspecto en general. Fuente: [13]. Muñoz, G., 2015.

El cultivo obtenido con el sistema de riego desarrollado es caracterizado por plantas de color verde más intenso, más fresco, de mayor tamaño y con fruto que evidencia un buen tamaño de mazorca y de grano, lo cual incrementa su precio al momento de la cosecha como se puede ver en la Fig. 12.

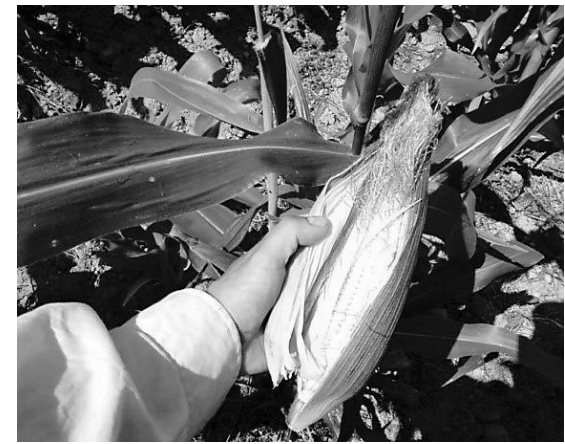

Fig. 12. Características de la mazorca con el sistema de riego autónomo. Fuente: [13]. Muñoz, G., 2015.

En la Fig. 13, es evidente el mayor tamaño de la mazorca recién colectada frente a un fruto de la cosecha anterior; ya que la semilla usada en el cultivo prototipo fue seleccionada de la cosecha anterior.

En este cultivo en particular, se observó que tuvo una mayor producción con un costo superior al del cultivo anterior; debido a los costos de implementación del sistema de riego, pero si se evalúa a profundidad el hecho de que el sistema se puede reutilizar para futuros cultivos, esta inversión se puede recuperar en el mediano plazo con el incremento de producción significativo, dependiendo de las condiciones del mercado; ya que se trata de un producto agrícola cuyos precios varían según la época del año.

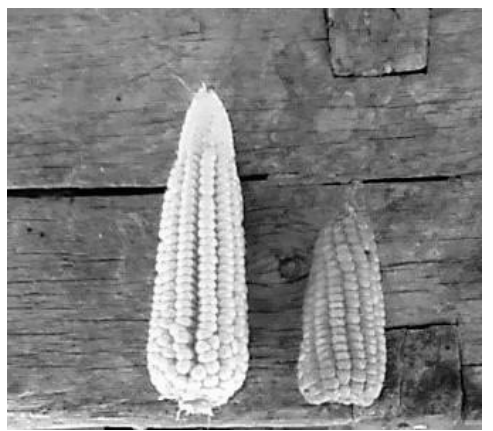

Fig. 13. Comparación de la mazorca del cultivo prototipo (izquierda) con la del cultivo anterior (derecha). Fuente: [13]. Muñoz, G., 2015.

\section{CONCLUSIONES}

El sistema de riego autónomo facilita el trabajo en la finca ya que reduce sustancialmente el tiempo utilizado en el riego del cultivo, siendo afectado solo por mantenimientos programados o de emergencia.

El hecho de usar un sistema de medición de humedad en lugar de uno temporizado elimina el sobre riego accidental, optimizando recursos y tiempo.

El diseño de la distribución de los aspersores en el área de cultivo, optimiza el uso del recurso hídrico con muy pocos puntos de sobre irrigación o sub irrigación que se tornan insignificantes al final.

El costo del sistema desarrollado es mucho menor que los que se encuentran en el mercado, ya que es asequible al agricultor colombiano con dispositivos electrónicos simples y de bajo costo.

El dispositivo se puede utilizar en diferentes métodos de riego, como el de cañón o la micro aspersión dependiendo de las necesidades del cultivo.

Para riego por goteo hay que tener muy en cuenta la necesidad de una cantidad muy grande de sensores, ya que se necesitaría uno por planta; lo que lo podría volver inviable un sistema de este tipo o hacer necesario un sistema más detallado para áreas de producción mayores. 
El incremento de producción obtenido de 5,87 veces muestra que es posible implementar este tipo de sistemas en pequeños y medianos cultivos con un retorno de capital a mediano plazo reflejado en un mejor valor del producto cosechado también.

El sistema puede ser mejorado con comunicación USB en lugar de RS232 y adquisición inalámbrica de datos como actualización tecnológica junto con la medición de variables complementarias como temperatura y $\mathrm{pH}$.

La implementación del sistema básico de riego automático mostró que pueden desarrollarse sistemas simples de bajo costo para la producción en pequeña escala en Colombia, capaces de aumentar en forma significativa la producción con una inversión relativamente pequeña y de retorno rápido a partir de los incrementos de producción obtenidos.

Para el mejoramiento de la producción agrícola en pequeña escala en Colombia, pueden ser desarrollados sistemas similares que sean modulares, simples, económicos y viables para el agricultor Colombiano; de tal forma, que pueden ser diseñados, implementados, amortizados y especializados poco a poco con inversiones razonables, obtenidas a partir de los beneficios económicos logrados con los incrementos de producción.

\section{AGRADECIMIENTOS}

Los Autores agradecen a los propietarios de la Finca La Reforma del municipio de Macheta, Cundinamarca, por facilitar los recursos para construcción e implementación del sistema experimental de riego en sus cultivos con el Ingeniero Guillermo Enrique Muñoz.

\section{REFERENCIAS BIBLIOGRÁFICAS}

[1] Blackmore, Simon. Agricultura de precisiónAP. Revista Nacional de Agricultura. Colombia. No. 949. 20-28, Junio 2007.

[2] Christy, C.D. Real-time measurement of soils attributes using on-the-go near infrared reflectance spectroscopy. Computers and
Electronics in Agriculture.Science Direct. 61, 10-19, 2008.

[3] Bakker, T. Wouters, H. Asselt, K. Bontsema, J. Müller, J. Van Straten, G. Tang, L. A Vision based row detection system for sugar beet. Computers and Electronics in Agriculture. Science Direct. 60, 87-95, 2008.

[4] García-Pérez, L. García-Alegre, M.C. Ribeiro, A. Guinea. D. An agent of behavior architecture for unmanned control of a farming vehicle. Computers and Electronics in Agriculture. Science Direct. 60, 39-48, 2008.

[5] De Bruin, S. Heuvelink, G.M.B. Brown, J.D. Propagation of positional measurement errors to agricultural field boundaries and associated costs. Computers and Electronics in Agriculture. Science Direct. 63, 245-256, 2008.

[6] Santé-Rivera, Inés. Crecente-Maseda, R. Miranda-Barrós, D. GIS-based planning support system for rural land-use allocation. Computers and Electronics in Agriculture. Science Direct. 63, 257-273, 2008.

[7] Jorgensen, R.N. Norremark, M. Sorensen, C.G. Andersen, N.A. Utilizing scripting language for unmanned and automated guided vehicles operating within row crops. Computers and Electronics in Agriculture. Science Direct. 62, 190-203, 2008.

[8] He, Y. Huang, M. García, A. Hernández, A. y Song, H. Prediction of soil macronutrients content using near-infrared spectroscopy. Computers and Electronics in Agriculture. Science Direct. 58, 144-153, 2007.

[9] Vellidis, G. Tucker, M. Perry, C. Kvien, C. y Bednarz, C. A real. time wireless smart sensor array for scheduling irrigation. Computers and Electronics in Agriculture. Science Direct. 61, 44-50, 2008.

[10] Robles, O. Playán, E. Cavero, J. Zapata, N. Assessing low-pressure solid-set sprinkler irrigation in maize, Agricultural Water Management, 191, 37-49, 2017. 
[11] Sheikhesmaeili, O. Montero, J. Laserna, S. Analysis of water application with semiportable big size sprinkler irrigation systems in semi-arid areas, Agricultural Water Management, 163, 275-284, 2016.

[12] Loghavi, M. y Behzadi, B. Development of a target oriented weed control system. Computers and Electronics in Agriculture. Science Direct. 63, 112-118, 2008.

[13] Ouazaa, S. Latorre, B. Burguete, J. y Zapata, $N$. Effect of intra-irrigation meteorological variability on seasonal center-pivot irrigation performance and corn yield. Agricultural Water Management 177. 201-214. 2016.

[14] Zapata, N. Salvador, R. Cavero, J. Lecina, S. López, C. Mantero, N. Anadón, R. y Playán, E. Field test o fan automatic controller for solid-set sprinkler irrigation. Irrig Sci 31. 1237 - 1249. 2012.

[15] Yingchao, L. Leikong. Baoyu, X. Taisheng, D. Shulin, H. Shaozhong, 2012 International Conference on Systems and Informatics ICSAI 2012. 397 -399. 2012.

[16] Muñoz, G. Diseño de un Sistema de riego autónomo para un cultivo pequeño de maíz en la finca La Reforma. [Trabajo de Grado para optar al título de Ingeniero en Automatización]. Bogotá, Colombia. Universidad de La Salle, 2015. 